\title{
Measurements of Black Hole Spins and Tests of Strong-Field General Relativity
}

\author{
Dimitrios Psaltis \\ Physics Department, University of Arizona, 1118 E. 4th St., Tucson, AZ 85721
}

\begin{abstract}
Fast variability studies of accreting black holes in the Galaxy offer us a unique opportunity to measure the spins of black holes and test the strong-field behavior of general relativity. In this review, I summarize the arguments often used in attempts of measuring the spins of black holes, concentrating on their theoretical foundations. I also argue that X-ray studies of accreting black holes will be able to provide in the future strong constraints on deviations from general relativity in the strong-field regime.
\end{abstract}

\section{INTRODUCTION}

Astrophysical black holes in general relativity are characterized by two quantities, their masses and spins, which determine uniquely the properties of their gravitational fields. As a result, both can, in principle, be measured by experiments involving test-particle orbits in their exterior spacetimes.

This approach has been very successful in measuring black-hole masses both in galactic systems (McClintock \& Remillard 2003) and in the centers of galaxies (Schödel et al. 2002). However, the imprint of the spins of black holes on their spacetimes, i.e., the dragging of inertial frames, is very weak at the large distances from the horizons, where the observed orbits reside. It is expected that the detection of gravitational waves from close, inspiraling compact objects with LIGO and LISA will allow for a complete mapping of the spacetimes of the objects and hence for the measurement of black-hole spins (see, e.g., Hughes 2003). However, only a small fraction of mostly supermassive black holes exist in the near universe in configurations that will allow such studies.

Most of the black holes we observe today are visible because they accrete matter from their companions or the surrounding medium. The intense $\mathrm{X}$-ray radiation we detect is generated in a region only a few Schwarzschild radii around the black-hole horizons. As a result, these $\mathrm{X}$-ray photons carry with them the signatures of the strong gravitational fields in which they are produced and hence information regarding the masses and spins of the black holes.

In this review, I discuss the potential of measuring black-hole spins and confirming the predictions of general relativity, using their rapid-variability properties. In particular, I concentrate on the various methods of inferring black-hole spins that are based on the observations of constant-frequency quasi-periodic oscillations from galactic black-hole binaries (QPOs; see Remillard, this volume). The frequencies of these QPOs depend very weakly on the observed X-ray flux and, for this reason, it is believed that they are determined mostly by gravity and not by the hydrodynamic properties of the accretion flows, such as their temperatures and densities.

\section{QPOS AND THE SPINS OF BLACK HOLES}

A number of different arguments have been used recently in the literature for inferring the spins of black holes from the frequencies of observed QPOs. In this section, I describe the theoretical foundations of three types of arguments that appear to depend the least on the specifics of theoretical models.

\section{The maximum QPO frequency}

In general relativity, the orbits of test particles around a spinning black hole are characterized by three frequencies: the azimuthal (Keplerian) frequency $f_{\phi}$, which is simply equal to the inverse of the orbital period; the (radial) epicyclic frequency $\kappa$, which for nearly-circular orbits is equal to the frequency of radial oscillations around the mean orbit; and the (vertical) epicyclic frequency $f_{\perp}$. All three frequencies depend on the mass and spin of the black hole, as well as on the radius of the orbit. 


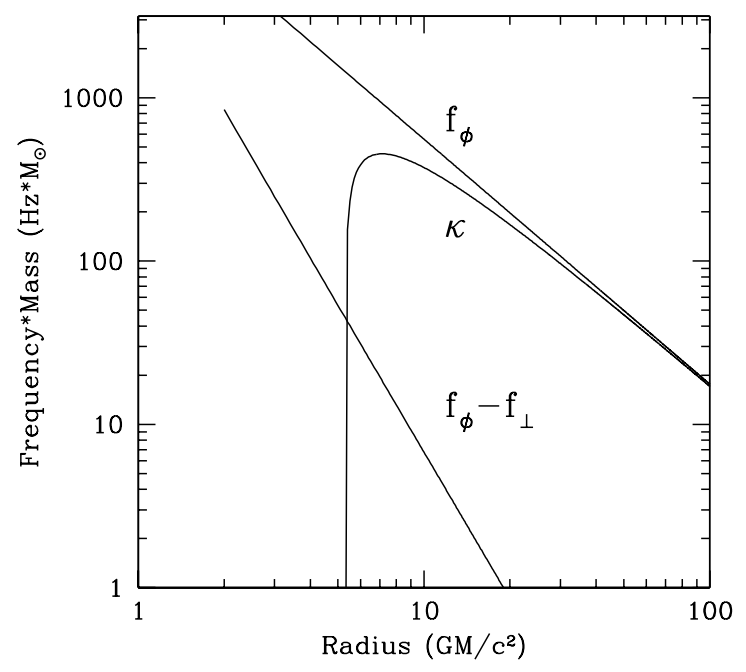

FIGURE 1. The azimuthal $\left(f_{\phi}\right)$, radial epicyclic $(\kappa)$, and vertical $\left(f_{\perp}\right)$ frequencies at different radii around a black hole with spin parameter $a / M=0.2$.

For each radius around the black hole, the azimuthal frequency $f_{\phi}$ is the highest among the three characteristic frequencies (see Fig. 1). Moreover, in general relativity, all circular orbits with radii smaller than that of the innermost stable circular orbit (ISCO) are unstable. This characteristic radius, at which the radial epicyclic frequency becomes zero, depends only on the mass $M$ and spin $a / M$ of the black hole and is given by (Bardeen et al. 1972)

$$
r_{\mathrm{ISCO}}=\left\{3+Z_{2} \mp\left[\left(3-Z_{1}\right)\left(3+Z_{1}+2 Z_{2}\right)\right]^{1 / 2}\right\} \frac{G M}{c^{2}}
$$

where

$$
\begin{aligned}
Z_{1}=1+ & \left(1-\frac{a^{2}}{M^{2}}\right)^{1 / 3} \\
& {\left[\left(1+\frac{a}{M}\right)^{1 / 3}+\left(1-\frac{a}{M}\right)^{1 / 3}\right], } \\
Z_{2}= & \left(3 \frac{a^{2}}{M^{2}}+Z_{1}^{2}\right)^{1 / 2},
\end{aligned}
$$

and the sign in equation (1) depends on whether the orbit is prograde or retrograde with respect to the blackhole spin. Because the azimuthal frequency decreases monotonically with radius,

$$
f_{\phi}(r)=\frac{(G M)^{1 / 2}}{2 \pi}\left[r^{3 / 2} \pm \frac{a}{M}\left(\frac{G M}{c^{2}}\right)^{3 / 2}\right]^{-1}
$$

it follows that stable circular orbits can exist only with azimuthal frequencies less than $f_{\phi}\left(r_{I S C O}\right)$. It is expected

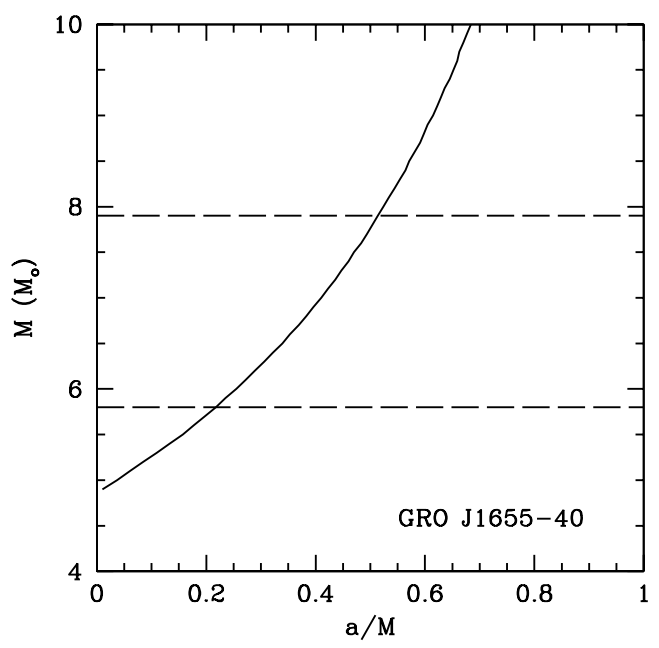

FIGURE 2. Dashed Lines: the dynamical measurement of the black-hole mass in the source GRO 1655-40. Solid Line: the minimum spin parameter $a / M$ for each black-hole mass, for which the observed $450 \mathrm{~Hz}$ QPO can be produced as a Keplerian frequency of a stable orbit. According to this argument, the black hole in GRO $1655-40$ is spinning with $a / M>0.2$ (after Strohmayer 2001a).

that for any model of quasi-periodic oscillations in accretion flows, the maximum frequency of any lowest-order, linear hydrodynamic mode in the accretion flow will also be smaller than the azimuthal Keplerian frequency evaluated at the innermost stable circular orbit, i.e., that

$$
f_{\mathrm{QPO}} \leq f_{\phi}\left(r_{\mathrm{ISCO}}\right)
$$

For a given observed QPO frequency, inequality (5) results in a minimum spin parameter $a / M$ for the black hole as a function of its mass $M$. This is illustrated in Fig. 2 for the black hole in GRO J1655-40 and the observed maximum QPO frequency of $450 \mathrm{~Hz}$ (see Strohmayer 2001a). The combination of inequality (5) (solid line) with the black-hole mass (dashed line) measured from observations of the binary orbit (Shabaz et al. 1999) provides a firm lower bound on the spin parameter for this black hole of $a / M \geq 0.3$ (Strohmayer 2001a).

The above argument provides the most modelindependent constraint on the spin of a black hole based on its rapid variability characteristics. Similar arguments have been used also in constraining the masses of neutron stars showing high-frequency QPOs (Miller et al. 1998) and of AGN exhibiting quasi-periodic variability (e.g., Iwasawa et al. 1998). They are general and model independent; however, they rely on two rather restrictive assumptions: $(i)$ that we are observing the lowest-order, linear modes in the accretion flows and (ii) that none of these modes occurs at a frequency higher than the local Keplerian frequency. Even though it is probably safe 


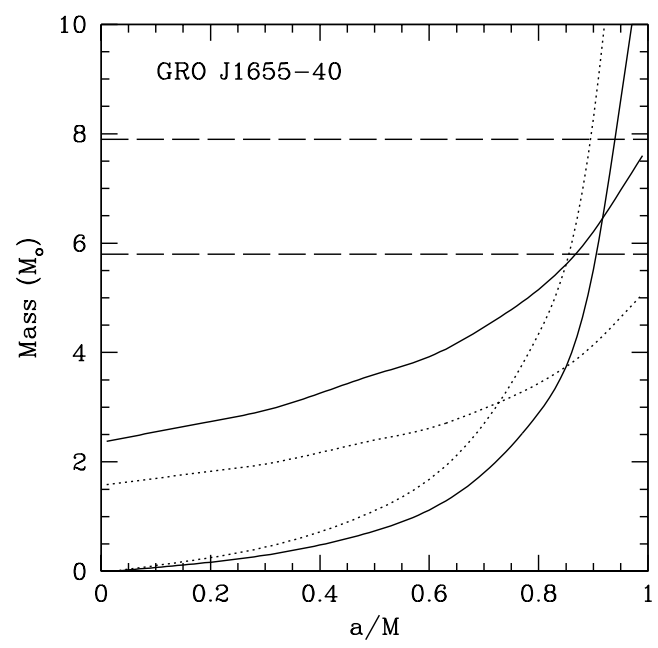

FIGURE 3. Dashed Lines: the dynamical measurement of the black-hole mass in the source GRO J1655-40. The intersection of the solid lines corresponds to the black-hole mass and spin for which the observed $450 \mathrm{~Hz}$ and $300 \mathrm{~Hz}$ QPOs are identified as the lowest-order $g$ and $c$ modes, whereas this identification is reversed in drawing the dotted lines. According to this explanation, the black-hole spin is $a / M \sim 0.9$ (after Wagoner et al. 2001).

to make the second assumption, the frequency ratios of constant-frequency QPOs in black-hole candidates (see below) cast serious doubts on the validity of the first assumption and hence on the generality of this argument.

\section{QPOs as Linear Modes}

A more accurate, albeit model dependent, measurement of the spin of a black hole can be achieved by identifying the two observed QPOs with the frequencies of particular linear global modes in the accretion flows.

The excitation and trapping of global modes in hydrodynamic flows around black holes, when the effects of magnetic fields and radiation forces have been neglected, has been studied extensively over the last twenty years (see Kato 2001 and references therein). In particular, three types of modes have been identified as potential sources of quasi-periodic variability:

- the $g$-modes: these are inertia-gravity modes that occur at a frequency $f_{\mathrm{g}} \simeq \kappa \pm m f_{\phi}$ (Perez et al. 1997; but see Li et al. 2003);

- the $p$-modes: these are inertia-acoustic modes and are not expected to produce significant modulation of the $\mathrm{X}$ ray flux (Ortega-Rodriguez et al. 2002);

- the $c$-modes: these are corrugation modes that occur at a frequency $f_{\mathrm{c}} \simeq f_{\phi}-f_{\perp}$ (Silbergleit et al. 2001).

The frequencies of all these modes depend primarily on the mass and spin of the black hole and weakly on the hydrodynamic properties of the accretion flows.

Identifying an observed QPO frequency with one of these modes leads to a relation between the values of black-hole mass and spin for which the observed frequency can be obtained. Identifying two observed QPO frequencies with two different modes leads to a single pair of black-hole mass and spin. This is illustrated in Fig. 3 for the $300 \mathrm{~Hz}$ and $450 \mathrm{~Hz}$ QPOs observed from the black hole in GRO J1655-40. The intersection of the dotted lines corresponds to the black-hole mass and spin for which the lowest-order $c$-mode frequency is $\simeq 300 \mathrm{~Hz}$ and the lowest-order $g$-mode frequency is $\simeq 450 \mathrm{~Hz}$. On the other hand, the intersection of the solid lines corresponds to the black-hole mass and spin for which the identification of the observed frequencies with the above modes has been interchanged. As long as this model is correct, there are no free parameters in obtaining these two pairs of values for the black-hole mass and spin!

The dynamical measurement of the mass of the black hole provides, in principle, an independent test of the validity of this argument. In the case of GRO J1655-40 (Fig. 3), the dynamically measured mass (area between the dashed lines) is consistent with one of the two masses inferred from the QPO identification and the corresponding spin parameter of the black hole is $a / M \simeq 0.9$ (Wagoner et al. 2001). The large value of the black-hole spin may be related to the fact that GRO J1655-40 is one of the few known microquasars in the galaxy.

\section{QPOs as Resonances}

Both of the arguments presented in the previous subsections rely on the assumption that the observed QPOs are generated at the frequencies of linear hydrodynamic modes of the accretion flows. However, in the three systems for which pairs of constant-frequency QPOs have been detected (including GRO J1655-40), the ratios of the frequencies of the QPOs are consistent with being equal to the ratios of small integers $(3 / 2$ and $5 / 3$; see Strohmayer 2001a, 2001b; Remillard et al. 2002). This property has led to the suggestion that the observed QPOs do not correspond to independent, linear modes but rather correspond to pairs of non-linear modes in resonance (Abramowicz \& Kluzniak 2001; see also Abramowicz, this volume).

In contrast to the trapping of global, linear modes in the accretion flows discussed earlier, in this interpretation, pairs of oscillatory modes attain high amplitudes at certain radii in the accretion flows where their frequencies have ratios equal to the ratios of small integers. Because the radius of resonance is a free parameter, 


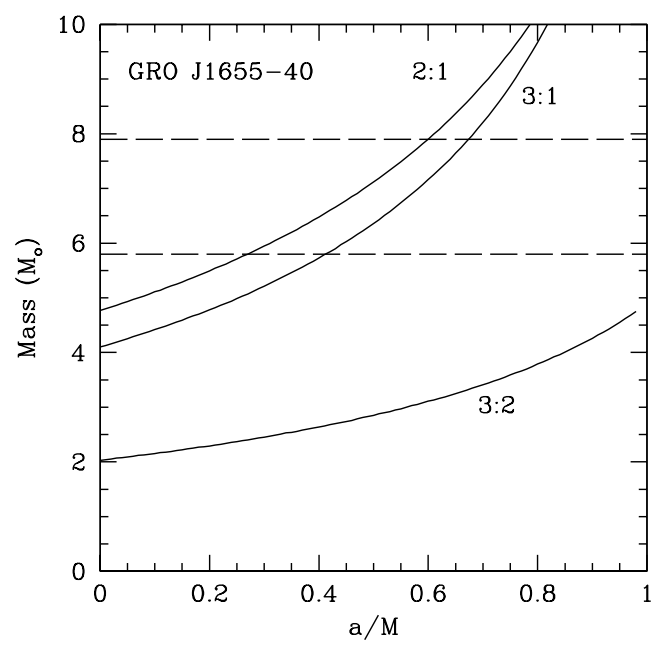

FIGURE 4. Dashed Lines: the dynamical measurement of the black-hole mass in the source GRO J1655-40. Solid Lines: the combination of black-hole mass and spin for which the observed $450 \mathrm{~Hz}$ and $300 \mathrm{~Hz}$ QPOs can be identified as a 2:1, 3:1, or 3:2 resonance between the Keplerian and radial epicyclic frequencies. According to this identification, the black-hole spin is $a / M \sim 0.2-0.7$ (after Abramowicz \& Kluzniak 2001).

the identification of two QPO peaks with the resonance between two frequencies does not lead to a single pair of values for the black-hole mass and spin but rather to a monoparametric family of possible values (the radius of resonance being the parameter). Moreover, additional freedom exists, at present, because different characteristic frequencies may be at resonance in various ways. This is illustrated in Fig. 4, where the observed $450 \mathrm{~Hz}$ and $300 \mathrm{~Hz}$ QPOs in the black hole in GRO J1655-40 are identified as a 2:1, 3:1, or 3:2 resonance between the azimuthal and radial epicyclic frequencies in the equatorial plane of the surrounding spacetime. Clearly, only the first two of the three alternatives result in black-hole masses consistent with the dynamical mass measurement of this source and, for those two alternatives, the inferred spin parameter of the black-hole is $a / M \sim 0.2-0.7$ (Abramowicz \& Kluzniak 2001; a larger spin is inferred if the resonance is assumed to occur between the vertical and radial epicyclic frequencies; see Abramowicz, this volume).

\section{Measuring Black-Hole Spins}

The above line of arguments makes clear the potential of measuring the spins of black holes using their rapid variability properties. Contrary to many other complex aspects of compact objects, nature has been kind enough to provide black holes with quasi-coherent X- ray flux modulation at nearly constant frequencies. This constancy strongly argues in favor of identifying the observed QPO frequencies with dynamical frequencies that depend only on the mass and spin of the black holes, making the inference of the latter only weakly model dependent. However, for a precise measurement of the spin of a black hole to be achieved, a number of important issues need to be resolved:

- Are linear, super-Keplerian modes possible in accretion disks? The least model-dependent arguments that may lead to a measurement of the spin of a black hole rely entirely on the assumption that such modes cannot exist.

- Are the observed QPO frequencies in ratios of small integer numbers? Non-linear coupling of modes appears impossible to neglect.

- What are the effects of magnetic fields on the frequencies of linear modes in accretion disks? Only a handful of calculations of linear modes have been performed when the effects of magnetic fields are not neglected (Gammie $\&$ Balbus 1994; Curry \& Pudritz 1995), even though it is thought that MHD turbulence provides the main reason why black holes accrete at the high observed rates.

- Why are there no QPOs in numerical simulations of MHD accretion disks? Current limitations in computer power allow only for simulations of geometrically thick disks in the absence of radiation cooling, both of which may be responsible for the lack of coherent large-scale oscillations (see, e.g., Hawley \& Krolik 2001, 2002; Armitage et al. 2001).

\section{STRONG-FIELD GRAVITY}

The identification of observed QPO frequencies with dynamical frequencies in the spacetimes of black holes provides the strongest evidence for the existence of black holes in the universe. Moreover, the potential of measuring the spins of nearly-maximally rotating black holes (as inferred, e.g., in Fig. 3) will have important implications for our understanding not only of the formation and evolution of black holes, but also of spin related phenomena such as jets and outflows. All the above carry the potential of providing the most constraining test of General Relativity in the strong-field regime todate.

\section{The Strongest Case for Black Holes}

Quasi-periodic oscillations in the X-ray flux of black holes retain phase coherence for many tens of cycles (see, e.g., Strohmayer 2001a, 2001b). Moreover, the QPOs discussed in the previous section have properties (e.g., coherence, amplitude, photon-energy depen- 
dence, etc.) that are reproducible and correlated with the spectral states of the sources. These have two direct consequences: $(i)$ the regions responsible for the quasiperiodic modulations have to be smaller than

$$
\begin{aligned}
\frac{R_{\mathrm{QPO}}}{R_{\mathrm{S}}} & \leq \frac{c^{3}}{G M f_{\mathrm{QPO}}} \\
& =96.3\left(\frac{f_{\mathrm{QPO}}}{300 \mathrm{~Hz}}\right)^{-1}\left(\frac{M}{7 M_{\odot}}\right)^{-1},
\end{aligned}
$$

where $R_{\mathrm{S}}$ is the Schwarzschild radius for an object of mass $M$ and $f_{\mathrm{QPO}}$ is the observed QPO frequency; and (ii) the modulating regions have to be arranged in an axisymmetric way around the black hole or the QPO properties would not be reproducible. The most probable configuration is the one in which the modulating region engulfs the central object and hence the observation of a $300 \mathrm{~Hz}$ QPO from the $\simeq 7 M_{\odot}$ source GRO J1655-40 strongly suggests that all seven solar masses are packed within less than one hundred Schwarzschild radii.

The above constraint can become significantly tighter if the observed QPO frequency is required to be lower than the azimuthal frequency at the radius where it is produced (see discussion in previous section). This is illustrated in Figure 5 for the case of the black hole in GRO J1655-40. Identifying the observed $450 \mathrm{~Hz}$ QPO with a frequency smaller than the azimuthal frequency at any radius implies that the central object is constrained to reside within $\sim 4-6$ Schwarzschild radii. This constraint is several orders of magnitude tighter than the one inferred from orbits of stars around the black hole in the center of the Milky Way (e.g., Schödel et al. (2002), where the central object is constrained to be at most $\sim 1000$ Schwarzschild radii.

\section{Testing Einstein Gravity}

A definitive proof for the existence of an event horizon around a compact object is by itself a test of the strong-field regime of general relativity. However, a large number of alternatives to general relativity allow also for the Schwarzschild solution and hence, in the limit of very slow rotation, are indistinguishable from it. The mapping of the spacetime of a rotating black hole using the properties of quasi-periodic oscillations appears to be the most promising way of testing directly the particular form of Einstein's theory of general relativity in the strong field regime.

There is very little experimental evidence today for the behavior of gravity in the strong-field regime (see, however, Damour \& Esposito-Farese 1993, 1996; DeDeo \& Psaltis 2003). This fact is illustrated in Fig. 6, where various probes and test of gravity theories are displayed on

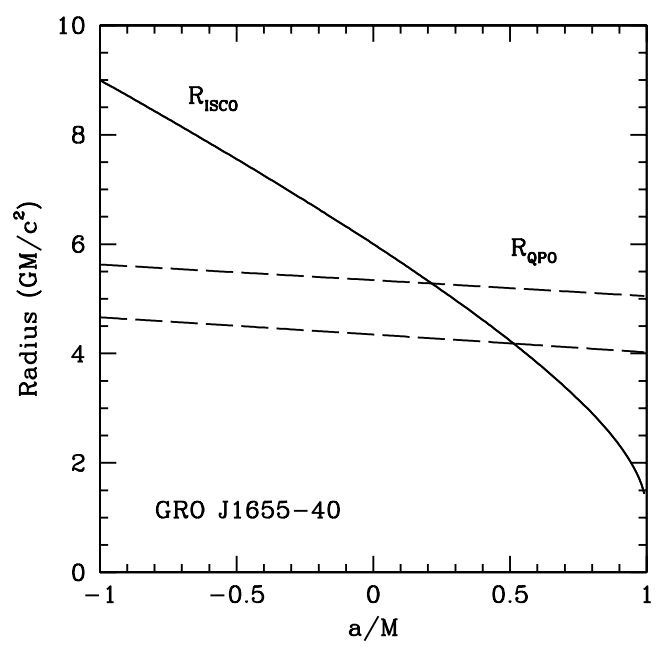

FIGURE 5. The dashed lines show the upper bound on the size of the $5.8-7.9 M_{\odot}$ compact object in GRO J1655-40, as a function of its spin, imposed by the requirement that the $450 \mathrm{~Hz}$ QPO frequency is at most the azimuthal frequency at some radius in the black-hole equatorial plane. This is the tightest observational constraint for the size of a black hole in the universe. The solid line shows the radius of the innermost stable circular orbit.

a parameter space consisting of the mass of the gravitating object and the potential $\left(\sim G M / R c^{2}\right)$ experienced by the test particles involved in the particular tests. Clearly, all current tests involve gravitational potentials that are at least five orders of magnitude weaker than the potential probed by QPO observations of black holes. These tests show that general relativity describes the weak-field regime of our universe to within at least $\sim 10^{-4}$ (as inferred, e.g., from tests of Brans-Dicke gravity; see Will 2001). As a result, deviations of order unity from the predictions of general relativity may appear in the strongfield regime, even though the corresponding deviations may be hidden from the weak-field tests.

Theories of gravity that are derived from an EinsteinHilbert action that is more general than that of general relativity are actively being considered as explanations of a number of puzzles in cosmology, such as the presence and magnitude of the cosmological constant (see, e.g., Carrol 2001). Theories with profound implications for the properties of solar-mass black holes will also affect the evolution of the universe at the time of nucleosynthesis (cf. Santiago et al. 1997). As a result, the properties of quasi-periodic black-hole variability may provide a probe of strong-field gravity that is complementary to cosmology. Analyses of such alternatives have been already been performed in the case of observables from neutron-star systems (Damour \& Esposito-Farese 1996; DeDeo \& Psaltis 2003). 


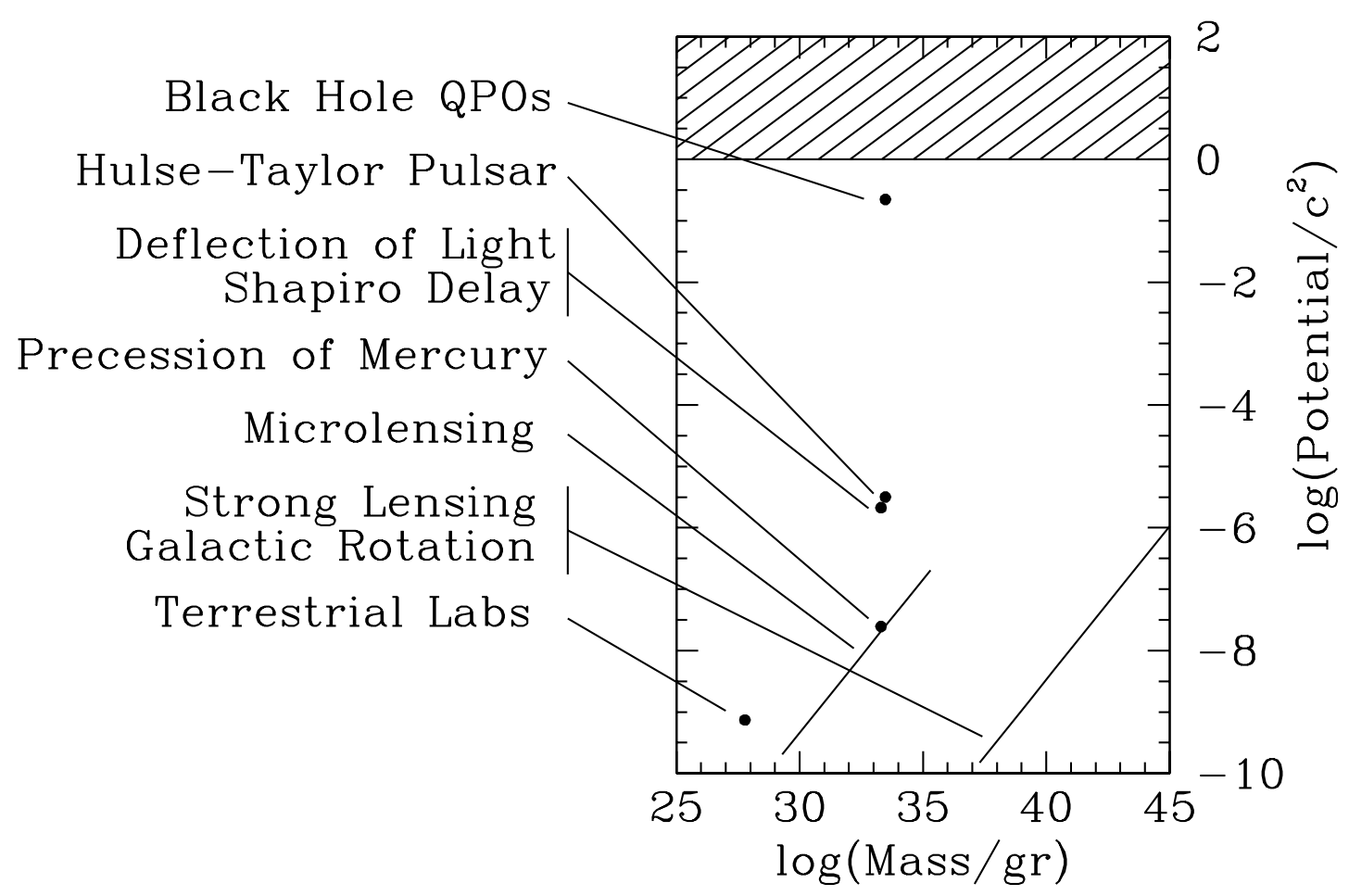

FIGURE 6. The gravitational potential that is probed by different tests of gravity plotted against the mass that generates it. Black-hole variability studies have the potential of testing gravity theories at fields that are up to five orders of magnitude stronger compared to any other tests.

\section{Constraining The Size of Large Extra Dimensions}

The complete theory of gravity may, of course, differ from general relativity not only in the form of the action from which it is derived by also in more profound ways, such as the number and scale of spacetime dimensions in which it is defined. The predicted properties and evolution of astrophysical black holes in such theories differs significantly from general relativity and hence may be directly testable.

Fig. 7 illustrates an example of constraining the size of a large extra dimension using the properties of the black hole in XTE J1118+480 (following Emparan et al. 2002). In theories with one large extra dimension, black holes evaporate significantly faster compared to what is predicted by general relativity. As a result, the inferred age of $240 \mathrm{Myr}$ for this $\sim 6.4 M_{\odot}$ black hole imposes an upper limit on the size of the extra dimension of $\sim 10^{-5} \mathrm{~m}$, which, albeit infinite compared to the Planck length, is at least an order of magnitude more stringent than the best upper limit obtained with table-top experiments on the sub-mm behavior of Newton's law of gravity.

\section{CONCLUSIONS}

Studies of black-hole variability probe the strongest field regime of gravity that is possible for observers outside the horizon of the black hole. They offer the potential of proving the existence of black holes in the universe, measuring their spins, and testing gravity theories in regimes that is unattainable by local experiments and can complement cosmological probes.

\section{ACKNOWLEDGMENTS}

It is a pleasure to thank Tomaso Belloni, Chi-Kwan Chan, Simon DeDeo, Mike Nowak, Feryal Özel, Martin Pessah, and Michiel van der Klis for all the discussions over the last several years that have helped me appreciate the various ways of using compact object variability in measuring black hole spins and in testing strong-field gravity. 


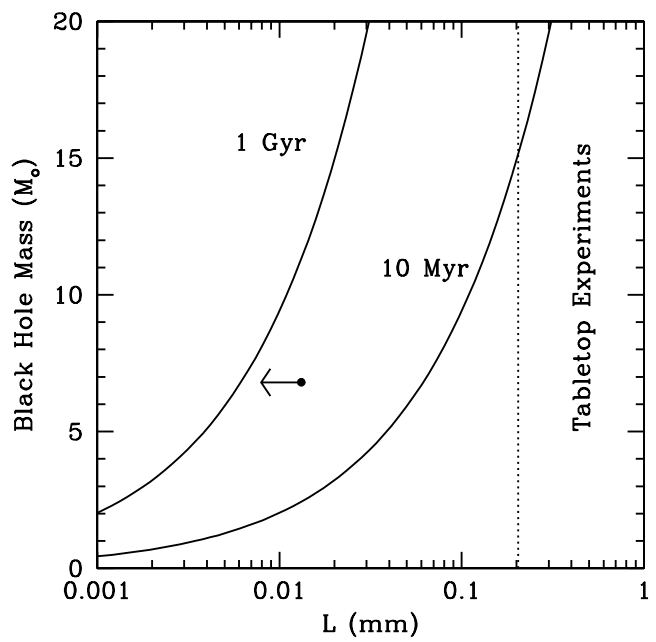

FIGURE 7. The solid lines are contours of constant lifetime for black-holes of stellar mass in theories with a large extra dimension, as a function of the size of the extra dimension. Table-top experiments provide a bound of $L \leq 0.2 \mathrm{~mm}$. The dot represents the upper bound on the size of the large extra dimension imposed by inferring an age of at least $240 \mathrm{Myr}$ for the $6.8 \pm 0.4 M_{\odot}$ black hole in XTE J1118+480 (after Emparan et al. 2002).

\section{REFERENCES}

1. Abramowicz, M. A. \& Kluźniak, W. 2001, A\&A, 374, L19

2. Armitage, P. J., Reynolds, C. S., \& Chiang, J. 2001, ApJ, 548,868

3. Bardeen, J. M., Press, W. H., \& Teukolsky, S. A. 1972, ApJ, 178, 347

4. Carroll, S. M., 2001, Living Rev. Relativity 4, 1 [Online article]: cited on 1 Feb 2004; http://www.livingreviews.org/lrr-2001-4

5. Curry, C. \& Pudritz, R. E. 1995, ApJ, 453, 697

6. Damour, T. \& Esposito-Farese, G. 1993, Phys. Rev. Let., 70,2220

7. . 1996, Phys. Rev. D, 54, 1474

8. Dedeo, S. \& Psaltis, D. 2003, Phys. Rev. Let., 90, 141101

9. Emparan, R., Garcia-Bellido, J., \& Kaloper, N. 2002, hep-th/0212132

10. Gammie, C. F. \& Balbus, S. A. 1994, MNRAS, 270, 138

11. Hawley, J. F. \& Krolik, J. H. 2001, ApJ, 548, 348

12. . 2002, ApJ, 566, 164

13. Houghes, S. A. 2003, Annals Phys., 303, 142

14. Iwasawa, K., Fabian, A. C., Brandt, W. N., Kunieda, H., Misaki, K., Terashima, Y., \& Reynolds, C. S. 1998, MNRAS, 295, L20

15. Kato, S. 2001, PASJ, 53, 1

16. Krolik, J. H. \& Hawley, J. F. 2002, ApJ, 573, 754

17. Li, L., Goodman, J., \& Narayan, R. 2003, ApJ, 593, 980

18. McClintock, J. E., \& Remillard, R. A. 2004, in Compact Stellar X-ray Sources, eds W.H.G. Lewin and M. van der Klis (Cambridge: University Press)

19. Miller, M. C., Lamb, F. K., \& Psaltis, D. 1998, ApJ, 508, 791
20. Ortega-Rodríguez, M., Silbergleit, A. S., \& Wagoner, R. V. 2002, ApJ, 567, 1043

21. Ogilvie, G. I. 1998, MNRAS, 297, 291

22. Perez, C. A., Silbergleit, A. S., Wagoner, R. V., \& Lehr, D. E. 1997, ApJ, 476, 589

23. Remillard, R. A., Muno, M. P., McClintock, J. E., \& Orosz, J. A. 2002, ApJ, 580, 1030

24. Santiago, D. I., Kalligas, D., \& Wagoner, R. V. 1997, Phys. Rev. D, 56, 7627

25. Schödel, R. et al. 2002, Nature, 419, 694

26. Shahbaz, T., van der Hooft, F., Casares, J., Charles, P. A., \& van Paradijs, J. 1999, MNRAS, 306, 89

27. Silbergleit, A. S., Wagoner, R. V., \& Ortega-Rodríguez, M. 2001, ApJ, 548, 335

28. Strohmayer, T. E. 2001a, ApJ, 552, L49

29. - 2001b, ApJ, 554, L169

30. Wagoner, R. V., Silbergleit, A. S., \& Ortega-Rodríguez, M. 2001, ApJ, 559, L25

31. Will, C.M., 2001, Living Rev. Relativity 4, 4 [Online article]: cited on $1 \mathrm{Feb} 2004$; http://www.livingreviews.org/lrr-2001-4 